\title{
Proyecto Recrea: una reflexión sobre la práctica educativa
}

\author{
REcrea Project: A reflection on educational practice
}

\author{
Yunuen Socorro Rangel ledezma • Renzo Eduardo Herrera Mendoza • David Manuel Arzola Franco
}

Yunuen Socorro Rangel Ledezma. Universidad Autónoma de Chihuahua/Centro de Investigación y Docencia, México. Es profesora-investigadora de tiempo completo en la UACH y profesorainvestigadora de horas en el CID. Es licenciada en Psicología y máster en Psicomotricidad. Ha laborado como docente en preescolar y primaria en el colegio particular Leonardo Da Vinci. Es integrante del Sistema Nacional de Investigadores en nivel Candidato desde el 2017 hasta la fecha. Correo electrónico: yrangel@uach.mx. ORCID: https://orcid.org/00000001-6419-1342.

Renzo Eduardo Herrera Mendoza. Centro de Investigación y Docencia, Chihuahua, México. Profesorinvestigador del programa de doctorado en Ciencias de la Educación del CID. Cuenta con estudios de Ingeniería Industrial, maestría en Dirección de Operaciones, maestría en Administración y doctorado en Administración Pública. Es evaluador de proyectos de emprendimiento, coach Ontológico y coach en Psicología Gestalt en Desarrollo Humano. Se dedica a impartir talleres de Desarrollo Humano enfocados a contribuir a la calidad de vida de las personas. Correo electrónico: renzo.herrera@cid.edu.mx. ORCID: https://orcid.org/0000-0001-6477-4476.

David Manuel Arzola Franco. Centro de Investigación y Docencia, Chihuahua, México. Miembro fundador del CID. A lo largo de 20 años ha participado en varios proyectos de investigación, enfocados a la educación básica. Es socio del Consejo Mexicano de Investigación Educativa (COMIE) y socio fundador de la Red de Investigadores Educativos Chihuahua (REDIECH). Se desempeñó por seis años como director de IE Revista de Investigación Educativa de la REDIECH (2010-2016). Actualmente

\section{Resumen}

El propósito de este trabajo es compartir las experiencias y reflexiones sobre un proyecto que se incorpora a la práctica docente en un programa de posgrado, con la pretensión de implementar estrategias innovadoras y generar reflexiones sobre las actividades cotidianas. La investigación tiene como base la incorporación a un proyecto denominado RECREA. El análisis involucra el trabajo desarrollado por un equipo de doce docentes, los cuales imparten materias diversas dentro de un programa de maestría en Educación. Se realizó una capacitación previa sobre el proyecto, así como indicaciones generales para generar las planeaciones, denominadas "andamios", tanto para el docente como para el alumno, así como los lineamientos y posibilidades de instrumentación didáctica para el trabajo en el aula. En el análisis se describe la manera en que la dinámica con el nuevo programa, la planeación e implementación de actividades, impactan en la cotidianidad de la práctica docente. En las conclusiones se advierte mejora en los procesos de comunicación entre los académicos, retroalimentación de avances, utilización de TIC y reflexión sobre las actividades cotidianas en clase.

Palabras clave: Buenas prácticas educativas, calidad docente, formación docente, práctica profesional.

Abstract
The purpose of this work is to share experiences on a teaching-incorpo-
rated project within a graduate program, with the aim of implementing
innovative strategies and generating reflections on daily activities. The
research is based on joining a project called RECREA. The analysis involves
the work developed by a team of twelve teachers, who teach various
subjects within a Master of Education program. Previous training on
the project was carried out, as well as general indications to produce the
plannings, named as "scaffolds", for both the teacher and the student;
as well as the guidelines and possibilities of didactic instrumentation
for work in the classroom. The analysis describes the way in which the


es coordinador del Cuerpo Académico de Política y Gestión en Educación y coordinador del doctorado en Ciencias de la Educación del CID. Correo electrónico: david.arzola@ cid.edu.mx. ORCID: https://orcid.org/00000003-2285-099X. dynamics with the new program, the planning and implementation of activities, impact the daily life of teaching practice. The conclusions show improvement in communication processes among academics, feedback on progress, use of ICTs and reflection on daily activities in class.

Keywords: Good teaching practices, teaching quality, teacher training, professional practice.

\section{INTRODUCCIÓN}

En la actualidad, la globalización y la forma de accionar para resolver las necesidades inmediatas del entorno hacen que el docente genere una automatización en su quehacer antes, durante y después de la impartición de un curso, es decir, se centra en el conocimiento previo y lo llega a realizar sin generar un análisis en cada una de las etapas de la docencia al momento de interactuar con los estudiantes. Al momento de generar una modificación en la rutina se hace un alto para pensar sobre las acciones realizadas, tratar de conjuntarlas con nueva información y, con ello, establecer actividades que se adapten a las nuevas necesidades presentes en su actuar.

Debido a lo anterior, el presente trabajo está enfocado en la reflexión sobre la práctica cotidiana del docente a partir de los procesos detonados por la aplicación de un proyecto denominado Red de Comunidades para la Renovación de la Enseñanza Aprendizaje en Educación Superior (RECREA), iniciado en el año 2017 por la Subsecretaría de Educación Superior de la Secretaría de Educación Pública (SEP), a través de dos dependencias: la Dirección General de Educación Superior para Profesionales de la Educación (DGESPE) y la Dirección General de Educación Superior Universitaria (DGESU).

El proyecto pretende que con la colaboración entre las universidades públicas estatales (UPEs) y las escuelas Normales urbanas y rurales públicas (ENP) de México se cree un adecuado desarrollo profesional docente, mediante la generación de procesos de análisis, reflexión, trabajo colaborativo, uso de metodologías innovadoras y estrategias pertinentes para la enseñanza-aprendizaje de los estudiantes (SEP, 2017). Es decir, el docente vaya más allá de su labor directa con los estudiantes, como establecer comunidades de aprendizaje en la realización, implementación, compartir el conocimiento de manera innovadora y consciente.

De acuerdo con los documentos generados en una plataforma digital creada para el trabajo de la Red de Comunidades para la Renovación de la Enseñanza Aprendizaje en Educación Superior (RECREA, 2017), el proyecto tiene el propósito de "promover una cultura de colaboración entre académicos para la innovación y mejora de sus prácticas docentes" (p. 1); se presenta como estrategia principal incorporar a la práctica educativa contenidos relacionados con el pensamiento complejo, el modelo 
por competencias, el uso de tecnologías de la información y comunicación (TIC), así como compartir experiencias en los procesos de enseñanza y aprendizaje.

A la vez, el trabajo se basa en un modelo de planeación pedagógica que se concretó en dos partes: a) "Andamio para la planificación de la docencia", generado por y para el docente que impartirá la secuencia didáctica establecida; el contenido se encuentra dividido en seis etapas: 1 . Contexto de la asignatura; 2 . Tareas y proyectos basados en el aprendizaje complejo; 3. Información teórica, procedimental y prácticas; 4. Apoyos y mediación, para la impartición de las actividades; 5 . Evaluación del desempeño, con opciones de rúbricas, listas de cotejo, diferentes tipos de valoración, y 6. Presentación a los estudiantes. La segunda parte del modelo es b) "Andamio para la presentación a los estudiantes", compuesto de las siguientes etapas: I. Presentación, de la materia, los casos a trabajar; II. Dispositivo de formación, las formas de interacción docente y estudiante en las diferentes etapas; III. Dispositivo de evaluación, basados en el pensamiento complejo y el desarrollo de competencias, con el establecimiento de metas claras y directas para conocer lo que el docente espera del estudiante, y IV. Referencias bibliográficas.

Para alcanzar tal propósito se generó una propuesta de acción en la que participaron diversos grupos de docentes, los cuales laboran en diferentes universidades públicas y escuelas Normales a lo largo del país, con la intención de compartir prácticas, problemáticas, alternativas y soluciones a diversas situaciones antes, durante y después de realizada la planeación en los “andamios" del docente y el estudiante.

El proyecto RECREA está encaminado a fomentar los procesos de transformación a través de tres ejes: a) un enfoque epistemológico sustentado en el pensamiento complejo y el desarrollo de competencias profesionales; b) la incorporación de los últimos avances de la investigación en el proceso y contenido de la enseñanza-aprendizaje, c) y el uso de tecnologías de la información y comunicación. Lo anterior mediante el método de la investigación-acción (Pedroza, 2017), con trabajo basado en problemas para diseñar estrategias adecuadas a cada contexto, mediante el uso del análisis y reflexión sobre el proceso de enseñanza y aprendizaje (Zambrano-Ramírez, 2016), es decir, mediante el pensamiento complejo.

El proyecto pone énfasis en la colaboración como una forma de detonar procesos de revaloración de los espacios de las aulas, para dar respuesta a las exigencias sociales y generar una identidad más sólida (Rodríguez, 2017). Los procesos participativos se caracterizan por impulsar el trabajo conjunto, así como el análisis crítico de los aspectos normativos y técnicos, los hábitos mecanicistas y la imagen estereotipada del proceso enseñanza-aprendizaje, elementos que contribuyen a la rutinización de las prácticas (López, 2003).

Por otra parte, la reflexión sobre la práctica docente genera cambios constantes y contribuye a establecer nuevas estrategias, enfocadas en las necesidades del contexto (Latapí, 2003), ya que sin esta la practica se convierte en un accionar monótono y hasta sin sentido; debido a que la reflexión conlleva a la autoevaluación permite estar 
consciente de si la dirección presente es la adecuada o es necesaria una autodirección para un adecuado desarrollo docente (Martínez-Izaguirre, Yániz-Álvarez y VillardónGalleg, 2018).

A finales del 2018 el Centro de Investigación y Docencia (CID), institución de posgrado ubicada en la capital del estado de Chihuahua, fue invitado por parte de la Coordinación de la Universidad Autónoma de Chihuahua (UACH) para formar parte de esta comunidad, en la cual también participaron docentes de la institución Benemérita y Centenaria Escuela Normal del Estado Profr. Luis Urías Belderráin. De esta manera los académicos del CID se involucraron en un proceso cuyos alcances se pretende documentar a través de este trabajo.

\section{DesarRollo}

Una vez que el proyecto se aplicó en otros espacios, se invitó a los docentes integrantes de la maestría en Educación del CID a participar en la comunidad generada por RECREA. Tras aceptar la invitación se generó una capacitación para dar a conocer y establecer las bases generales de operación del proyecto. El curso de capacitación se implementó con una duración de 40 horas, divididas en tres fases: una primera parte en modo presencial durante 20 horas, distribuidas en el transcurso de una semana, con la integración de trabajo independiente; la segunda parte consistió en trabajo independiente por parte del colectivo, dando como opciones la realización del "andamio" individual o en colectivo; la tercera parte fue dividida en dos sesiones de seguimiento de 10 horas. Las últimas horas del curso se dedicaron a consensuar y revisar los trabajos independientes en dos sesiones, todo esto realizado con el acompañamiento de docentes de la UACH y personal de la Escuela Normal del Estado.

Se establecieron trabajos de planeación mediante la denominación "Andamio para el docente" y "Andamio para el estudiante", lo considerado de manera anterior como una planeación; fueron aplicados en el periodo correspondiente, inmediatamente después de terminada la capacitación presencial. Durante este tiempo no se presenció seguimiento por parte de las instituciones de capacitación, sin embargo se definió por parte del CID la generación de reuniones periódicas por academia, dependiendo de las necesidades y la disposición de los docentes. Una vez terminado el periodo, se realizó una reunión colegiada de análisis, para la evaluación de los trabajos realizados antes, durante y después de la generación de los “andamios", según lo sugerido dentro del proyecto.

Para el presente trabajo se toma en cuenta la reflexión docente, otra forma de evaluación, ya que en México los trabajos de investigación suelen ser más estadísticos, sin tomar en cuenta el contexto donde se encuentra inmerso (Olivos, 2018), con la falta de lo verdaderamente importante para el desarrollo y progreso de la enseñanza: darse cuenta de lo que se realiza de manera personal y comunitaria, y cuáles de estas acciones ayudan en la contribución de una adecuada implementación de acciones en 
el día a día (Darling-Hammond y McLaughlin, 2003). La calidad de un docente es más que solo ir a generar el proceso enseñanza-aprendizaje, consiste en la combinación de personalidad, habilidades, conocimientos, actitudes, pedagogía del contenido, comprensión de sus estudiantes, disposición de ayuda, distribución de ideas, capacidad adaptativa (Darling-Hammond, 2013).

El trabajo general se llevó a cabo a través de la investigación-acción, con la intervención activa de los sujetos involucrados en cada una de las fases del proyecto mediante un análisis y reflexión constante. El ciclo de investigación concluyó con un proceso de autoevaluación a través de la técnica de grupos de enfoque o discusión, para revisión de experiencias donde se generó material audiograbado, que posteriormente fue utilizado como insumo para construir el informe de investigación con los académicos participantes del CID.

El análisis de la información del presente trabajo se realizó a partir del material empírico recabado en el grupo de discusión, que sirve como base para la recuperación de experiencias (Sisto, 2008). Los diálogos presentados se manejan con el formato de citas textuales, la información entre paréntesis procede de los datos de audiograbaciones, por ejemplo: (1:53 0:05:49.65 [0:00:36.11]); la nomenclatura se deriva del programa computacional utilizado para el análisis de la información.

\section{ANÁLISIS DE RESULTADOS}

Para generar este apartado de una manera práctica con relación a lo recabado durante las audiograbaciones generadas, para el conocimiento de los análisis y reflexiones de los propios docentes, se toman en cuenta los comentarios relacionados con las siguientes categorías: estrategias de trabajo colaborativo; generación de "andamios", e incorporación de recursos tecnológicos a la práctica docente.

El trabajo colaborativo consiste en las formas, alcances y comunicación entre los docentes al momento de generar un mismo "andamio", la generación de "andamios" sobre los conocimientos a trasmitir y la manera más adecuada para hacerlo llegar a los estudiantes con el manejo del pensamiento complejo, y la incorporación de recursos tecnológicos a la práctica docente; al momento de realizar el "andamio" cada docente se hace consciente de las herramientas que domina y cuales tiene como área de oportunidad para desarrollarlas y con ello utilizarlas de la mejor manera en su desempeño; esto también involucra las evaluaciones sobre la adquisición del conocimiento, en la búsqueda de hacerlo de manera uniforme de acuerdo a las necesidades del nivel académico y el grupo a trabajar.

El contexto influye en la interacción social e individual, las características personales e integración como participante de trabajo colaborativo, el ambiente; esto influye en los procesos de preparación, enseñanza y análisis, para lograr procesos significativos, los cuales son de suma importancia dentro de una institución educativa (Márquez, 2019). 
Con relación a las estrategias de trabajo colaborativo, de manera colectiva se propuso abrir academias por área hasta por materia, según se vio la necesidad de trabajar, con la intención de integrar conocimientos y compartir experiencias; dichas academias se trabajaron de manera constante, con reuniones de manera determinada, no solo en la generación del "andamio" sino al momento de la aplicación, mediante análisis y reflexión en colectivo sobre la forma de aplicación de estrategias y posibilidades de modificación de acuerdo con las características de los estudiantes y del docente a impartir.

Las cosas que yo le noté que fue bueno, es trabajar en academia, ya trabajábamos, pero, por ejemplo, trabajar juntos, planeando las actividades, las sesiones, innovar, implementar nuevas estrategias, revisar los materiales a utilizar en el curso, todo eso fue algo bueno, porque lo estuvimos haciendo continuamente, nos daba más claridad al ir a las sesiones con los alumnos, más seguridad (0:04:51.30 [0:00:40.72]).

... como que cada año que cumple uno se vuelve rutinario y se acomoda uno, al cabo ya vi eso, me compartieron otros lo que ya hicieron y me iba a acomodando, y este proceso me representó un alto en el camino de reflexión de aprendizaje, no nuevo, pero sí que me invita, que no lo sabemos todo, que tenemos que aprender de los demás, los compañeros para mí representaron un apoyo muy significativo, cuando en el trabajo de academia ponen al servicio de los demás su conocimiento, su experiencia, porque la experiencia se convierte en sabiduría (2:11:15.71 [0:01:06.50]).

Los espacios para reflexionar y debatir sobre los conocimientos aplicados y adquiridos durante el proceso, como menciona Rodríguez (2017), contribuyen a la mejora de las prácticas pedagógicas, ya que se analizan las rutinas de cada participante y se implementan estrategias para transitar de una práctica tradicional aislada a una colaborativa e innovadora, con preocupación por generar condiciones nuevas para los docentes ya que contribuye en la generación de una práctica asertiva.

El apoyo a la práctica reflexiva no existe sin una acción compleja, así como el análisis de la propia acción (Perrenoud, 2011). Sin embargo, se debe de hacer consciente de la situación individual en el proceso de reflexión para identificar sus habilidades, conocimientos, competencias de la realidad que le rodea y mejorar la práctica docente antes, durante y después de aplicar la planeación (Márquez, 2019); la acción reflexiva se puede realizar de manera individual o colaborativa, esta última consiste en una combinación de ambas, ya que para presentar el análisis en un colectivo primero debe de ser personal, con una conciencia completa de lo que se es y lo realizado en la práctica docente.

Por otra parte está la experiencia de los docentes que participaron en el programa pero no se integraron en alguno de los equipos, su reflexión queda de manera individual, vieron la imperiosa necesidad de investigar, buscar e interpretar de manera independiente lo solicitado en los formatos de planeación, así como establecer cambios en la rutina para el trabajo de la asignatura establecida; esto trajo el resultado que se expresa a continuación: 
Yo no lo concluí, le di hasta donde le di porque yo me quedé solo, no tuve la oportunidad de trabajar en conjunto, lo poco que entendí lo trabajé así. A veces siento que son esfuerzos que no van a tener un eco, no sé por qué siento que estamos haciendo como un requisito o algo que tenemos que cumplir, sabiendo que sí es muy importante (0:06:22.13 [0:00:32.44]).

El momento de participar en compartir experiencias y escuchar las de los compañeros es cuando se participa en esa comunidad y trabajo colaborativo, tomar las reflexiones de los demás e incorporarlas en un futuro de accionar docente consecuente.

Otro de los grandes temas en la generación de los "andamios" es la planeación o preparación de una clase, la cual es considerada una acción imprescindible para todo docente, por ello existe la necesidad de dedicación de tiempo; según esta se garantiza un éxito o fracaso en la transmisión de conocimientos, se toma en cuenta la forma de enseñanza del docente y la forma de aprendizaje del estudiante para lograr un adecuada interacción, sin embargo, para adquirir conciencia de los obstáculos presentes o los cambios necesarios para lograr el objetivo se requiere de un análisis constante de la práctica (Reyes-Salvador, 2017).

Con relación a la generación de los "andamios" (planeaciones) del docente y del estudiante, esta actividad fue propuesta en el área de investigación para complementar materiales y compartir información relacionada con sus temáticas; los participantes hacen una crítica al sesgo tecnicista del proyecto RECREA y a la formación misma de los docentes, que ponen especial énfasis en los formatos de planeación y en la exactitud de su diseño mas no en el contenido, ni en el contexto, ni en las necesidades de los alumnos.

La planeación depende de la maestría pedagógica, la creatividad e innovación de quien la genera, esto conlleva el manejo de la información, los contenidos; en niveles superiores se integran lo académico, lo laboral, la investigación y la extensión para una adecuada impartición; por ello, el tipo de planeación de clase difiere con el contenido de esta, a pesar de que ante los mismos estudiantes luzcan distintas (Espinoza, 2019). A pesar de que sea la misma materia, la personalidad del docente influye en la forma de planear, utilizar las TIC, las actividades y hasta la evaluación; aún trabajando de manera colaborativa, cada docente deja sus aprendizajes previos en la aplicación de esta.

...la obsesión por la planeación nos las injertaron en la Normal, de ahí la critica a RECREA; recuerdo, en la formación de la Normal, lo cierto es que el énfasis no estaba en la reflexión sobre la práctica, los contenidos y las necesidades de los alumnos, el énfasis estaba en el rigor en el cual se planeaba y en la exactitud con la cual se diseñaban las secuencias didácticas (1:03:12.37 [0:00:49.96]).

A pesar de los problemas que se identificaron en la propuesta de planeación, los participantes reconocen la importancia de la participación en el proyecto como una forma de generar desequilibrio y con ello dedicar tiempo para establecer una nueva dinámica, debido a la necesidad de buscar opciones pedagógicas congruentes con 
las demandas al momento de relacionar la asignatura a impartir con el proyecto y las necesidades de los estudiantes.

El desequilibrio que se genera al reflexionar es una combinación del ámbito cognitivo y emocional, mediante la sensación de incomodidad y desconcierto, siendo la parte emocional que genera el análisis cognitivo sobre incógnitas presentes como: ¿qué se está realizando?, ¿cómo?, ¿cuál es el nivel de compromiso al realizarlo?, ¿la finalidad y la forma serán las correctas? (Perrenoud, 2011). Un ejemplo de esto es el siguiente comentario y la forma de valorar la parte reflexiva que aportó a la práctica docente:

...obligarnos a entrar en ese cajón causó cierto desequilibrio porque nos movió de la rutina que llevamos cotidianamente, prácticamente yo ya sé de qué trata el seminario que va a venir, cuáles son los contenidos que voy a tratar, etcétera; entrar en sí a un proceso de planeación, por estructurado que sea, obligó a reflexionar la práctica que estamos haciendo (0:49:26.74 [0:00:39.99]).

La idea de los docentes de fortalecer los procesos de planeación se concretó en un trabajo minucioso en el cual se describen los procesos de enseñanza y aprendizaje, las estrategias, actividades, tareas; los diversos niveles de complejidad, objetivos, tipos de apoyo necesarios para el diseño de las asignaturas; se compartieron elementos claves para ubicar al estudiante en el contexto general del curso, el perfil de egreso, competencias y didácticas para las temáticas; es decir, tener una guía con la flexibilidad de accionar con la constante presencia del objetivo de aprendizaje (Morales, 2018).

Lo anterior genera reflexión de los cambios a realizar en la planeación, debido a que es considerada como una preparación importante para los docentes, así como una combinación teórico-metodológica para la práctica docente, sin embargo, un proceso necesario para la actualización de nuevas formas de aprendizaje (Reyes-Salvador, 2017); aunque suele ser visto como solo un cambio de formato, otros docentes sí se toman el tiempo para generar la reflexión necesaria para mejorar su desempeño en el proceso enseñanza-aprendizaje.

De acuerdo con los testimonios recabados se ve reflejado un incremento en el uso de las tecnologías de la información y comunicación para trabajar con los grupos, en el establecimiento de la forma de entrega, así como lo que se esperaba del grupo en general y particular desde el inicio de la clase; aunque a algunos docentes se les dificultó el cambio al uso de las tecnologías de la información, es decir, el proceso de actualizarse, innovar, activarse en esa forma de trabajo, por lo tanto, buscaron diversas formas de trabajar a distancia como complemento del proyecto, es decir, se generó la necesidad de un cambio y adaptación a los procesos planteados, la mayoría en colectivo, para la impartición de clases; la creatividad y adaptación se encontraban presentes como parte de cubrir ese requerimiento.

Como mencionan Rizzo y Pérez (2018), con las TIC hay que ir más allá de solo utilizarlas, sino saber la forma propicia para adecuar y optimizar la información, mediante la comprensión, el uso certero, socializarla con quienes se trabaja para generar 
una adecuada aplicación de estas. Es decir, mejoran y amplían las posibilidades de transmisión de conocimiento en el entorno educativo, y más al momento de dejar de resistirse en su utilización a favor y como un recurso útil en la función docente (Martínez-Izaguirre, Yániz-Álvarez y Villardón-Gallego, 2018). Se percibe de manera fácil y directa en los siguientes comentarios:

Generó inquietud para trabajar con TIC, y en muchos casos se concretaron trabajos en plataforma que no estaban presentes en la necesidad del CID; grosso modo me parece que el proyecto RECREA nos movió (0:52:43.18 [0:00:20.08]).

$\mathrm{Al}$ principio que hablaban de redes y las TIC, estoy en pañales en eso [...] El trayecto formativo iba más rápido que lo que yo aprendía de redes en el curso, entonces me inventé la forma de comunicarme con los muchachos, esa fue mi innovación [0:41:34.60 (0:00:50.94)].

Con los años de docencia se adquiere una capacidad para tomar decisiones sobre la marcha y modificar las actividades (Valencia, 2019). Así mismo se generó una reflexión de si realmente se encuentra presente la rutina, si se hace de manera adecuada, hasta llegar al punto de analizar el tipo y la forma de mejora a trabajar dentro del proyecto para renovar la práctica docente; durante ese proceso también se analiza la generación de actualización en las formas de aprendizaje de las generaciones que cambian poco a poco de acuerdo a como el contexto se va modificando.

\section{RefLEXIONES finALES}

En la práctica se manifestó una constante comunicación entre los integrantes del equipo del área, ya que se encontraban en diálogo permanente; durante las reuniones establecidas, sobre los efectos de la planeación en cada grupo, clarificaban dudas y modificaban las estrategias según el contexto y las necesidades de los estudiantes. De acuerdo con los testimonios recabados, el trabajo en equipo coadyuva en la construcción de una visión crítica sobre la práctica docente, revaloriza las acciones, genera o regenera ideas y contribuye en la atención de los estudiantes.

Debido a lo anterior, se puede concluir que participar en un proyecto como RECREA genera procesos de reflexión y análisis, debido al reacomodo entre la información nueva, la previa y la práctica cotidiana del docente, mediante el conocimiento de debilidades y fortalezas, de sus compañeros y propias, dentro del trabajo colaborativo. Se encuentra también como una ventaja incorporar en las dinámicas de las clases el uso de nuevas tecnologías, adaptándose para el manejo adecuado de la información y la interacción entre los estudiantes y el docente.

Adaptarse a un modelo de planeación como el denominado "andamio" para el docente y para el estudiante, dentro del proyecto, obligó al colectivo a revisar con mayor profundidad las actividades cotidianas y el seguimiento de estas antes, al momento de plantear las actividades, durante, al establecer las estrategias y la forma de accionar y recibir la información por parte de los estudiantes, y después de la clase, mediante análisis y reflexiones de la práctica docente de manera individual y colectiva. 
El equipo encontró en el trabajo colectivo una forma de romper la rutina, participar del diálogo con sus compañeros y retroalimentar el proceso al compartir las diversas formas de trabajo; generó una dinámica progresiva, en la que se exponían problemas y se aclaraban dudas; posteriormente se aplicaban y se volvían a poner a trabajar sobre las estrategias y materiales a trabajar.

El cambio dentro de la planeación con uso de actividades para desarrollar el pensamiento complejo rompe la rutina en la forma de planear, debido al movimiento de impartir conocimientos y habilidades a los estudiantes, por la manera de plantear y utilizar.

$\mathrm{Al}$ momento de recibir formas nuevas de trabajar se genera un análisis de las cosas que se han realizado, cómo, para qué, a quién va dirigido, y si aún son efectivas para los cambios en el contexto; una vez terminado el análisis, recibida la nueva forma de trabajar, se genera una adaptación de conocimientos, se integran estrategias que se ven de utilidad, se implementan; al finalizar conllevan a una reflexión de un antes y un después, para poder utilizar los elementos útiles y generar flexibilidad al recibir información que complemente la interacción.

Se generó un enriquecimiento de las buenas prácticas, así como una comunidad colaboradora y crítica al interior de la institución, debido a la participación en los grupos generados como apoyo para ingresar las estrategias en los diferentes grupos.

\section{REFERENCIAS}

Darling-Hammond, L. (2013). Getting teacher evaluation right. What really matters for effectiveness and improvement. Nueva York: Teacher College Press.

Darling-Hammond, L., y McLaughlin, M. (2003). El desarrollo profesional de los maestros. Nuevas estrategias y políticas de apoyo. Ciudad de México: SEP.

Espinoza, E. E. (2019). Planeación microcurricular desde la interdisciplinariedad en las carreras docentes. Revista Metropolitana de Ciencias Aplicadas, 2(3), 198-213.

Latapí Sarre, P. (2003). ¿Cómo aprenden los maestros? Conferencia magistral en el XXXV aniversario de la Escuela Normal Superior del Estado de México. Toluca: Secretaría de Educación Pública.

López, S. (2003). Construcción sociocultural de la profesionalidad docente: estudio de casos de profesores comprometidos con un proyecto educativo [Tesis doctoral]. Universitat de Valencia. Recuperado de: http://tdx.cat/bitstream/handle/10803/9647/lopez.pdf ?sequence=1.

Márquez, M. E. (2019). El trabajo colaborativo: una oportunidad para el desarrollo del pensamiento práctico del profesional reflexivo. Revista Scientific, 4(11), 360-379.

Martínez-Izaguirre, M., Yániz-Álvarez, C. y Villardón-Gallego, L. (2018). Autoevaluación y reflexión docente para la mejora de la competencia profesional del profesorado en la sociedad del conocimiento. Revista de Educación a Distancia (RED), (56), 1-30. DOI: http:/ / dx.doi.org/10.6018/red/56/10.

Morales, R. E. (2018). La planeación de la enseñanza-aprendizaje, competencia que fortalece el perfil docente. RIDE. Revista Iberoamericana para la Investigación y el Desarrollo Educativo, 8(16), 311-334. DOI: https://doi.org/10.23913/ride.v8i16.343.

Olivos, T. M. (2018). La evaluación docente en la universidad: visiones de los alumnos. REICE. Revista Iberoamericana sobre Calidad, Eficacia y Cambio en Educación, 16(3), 87-102. 
Pedroza, R. (2017). Proyecto de investigación de las comunidades de aprendizaje para la renovación de los procesos de enseñanza y aprendizaje de la educación superior. Recuperado de: https://promepca. sep.gob.mx/solicitudesca/login.aspx.

Perrenoud, P. (2011). Desarrollar la práctica reflexiva en el oficio de enseñar. Profesionalización y razón pedagógica (4a. reimp.). Barcelona, España: Graó.

ReCrea [Red de Comunidades para la Renovación de la Enseñanza Aprendizaje en Educación Superior] (2017). Seminario RECREA. Recuperado de: http:/ / cursos.acet-latinoamerica.org/ recrea/course/view.php?id $=21$.

Reyes-Salvador, J. (2017). La planeación de clase; una tarea fundamental en el trabajo docente. Maestro y Sociedad, 14(1), 87-96.

Rizzo, F., y Pérez, A. (2018). Importancia del uso de las Tics en los docentes. Espirales Revista Multidisciplinaria de Investigación, 2(23), 43-48.

Rodríguez, L. G. M. (2017). La construcción de una identidad docente, ¿un desafío para la política educativa? Revista Exitus, 3(1), 75-87.

Sánchez, J. T. G. (2017). Cultura de trabajo en equipo del personal docente de educación media general. Cienciamatria, 3(4), 35-48.

SEP [Secretaría de Educación Pública] (2017). Red de comunidades para la renovación de la enseñanzaaprendizaje en educación superior. Recuperado de: http://cursos.acetlatinoamerica.org/recrea/ $\mathrm{mod} /$ lesson $/$ view.php?id=1\& pageid=1\&startlastseen $=$ no.

Sisto, V. (2008). La investigación como una aventura de producción dialógica: la relación con el otro y los criterios de validación en la metodología cualitativa contemporánea. Psicoperspectivas, (7), 114-136.

Valencia Velásquez, F. (2019). Reflexión estilos de práctica docente. Recuperado de: https://docplayer.es/amp/169936755-Reflexion-estilos-de-practica-docente-grupo-1-la-profesiondocente-entre-la-reflexion-y-la-critica.html.

Velásquez, A. C. (2016). Iniciar la educación en ciudadanía desde el preescolar mediante rutinas de pensamiento. Infancias Imágenes, 15(1), 41-58.

Zambrano-Ramírez, J. (2016). Aprendizaje complejo en la educación superior ecuatoriana. Revista Ciencia UNEMI, 19(21), 158-167. Recuperado de: http://www.academia. edu/28940752/Aprendizaje_complejo_en_la_educaci\%C3\%B3n_superior_ecuatoriana.

Cómo citar este artículo:

Rangel Ledezma, Y. S., Herrera Mendoza, R: E., y Arzola Franco, D. M. (2020). Proyecto ReCrea: una reflexión sobre la práctica educativa. RECIE. Revista Electrónica Científica de Investigación Educativa, 5(1), pp. 191 -201. doi: doi.org/10.33010/recie.v5i1.1003. 